\title{
Serum intercellular adhesion molecule-1 (ICAM-1) and high-sensitivity (hs) C-reactive protein (CRP) in adolescent females: physical activity and dietary influences
}

\author{
E. Alshammari ${ }^{1}$, S. Shafi ${ }^{1}$, J. Nurmi-Lawton ${ }^{1}$, S. A. Lanham-New ${ }^{1}$ and G. A. A. Ferns ${ }^{1,2}$ \\ ${ }^{1}$ Faculty of Health and Medical Sciences, University of Surrey, Guildford, UK and ${ }^{2}$ Postgraduate Medical School, Daphne \\ Jackson Road, Guildford, Surrey, UK
}

CRP is an inflammatory marker that has been shown to be affected by dietary factors. Recent investigations suggest that physical activity (PA) may be associated with reduced serum CRP levels ${ }^{(1)}$. Intercellular adhesion molecule-1 (ICAM-1) is a trans-membrane glycoprotein that plays a key role in leucocyte migration and activation. ICAM-1 is up regulated on activated endothelial cells and its soluble form (sICAM-1) is increased in athletes ${ }^{(2)}$. Intense physical training ${ }^{(3)}$ and dietary factors ${ }^{(4)}$ may also effect its serum concentrations, although confounding effects of diet and adiposity have not been taken into account. Furthermore, many of these studies have been conducted in adults, in whom the onset of atherosclerosis may be a further confounding issue.

The aim was to assess whether serum sICAM-1 and hsCRP differ between physically-active female gymnasts and inactive females who were part of a 3-year longitudinal bone investigation ${ }^{(5)}$. A further aim was to assess the association between these two variables and BMI, dietary intake and PA. Healthy girls, including twenty-five competitive gymnasts and nineteen healthy sedentary individuals aged $8-17$ years $(13.0 \pm 0.7$ years; $12.0 \pm 0.5$ years $)$ respectively, were recruited. Human sICAM-1 and hsCRP were measured using commercial ELISA.

Serum sICAM (mg/L) was significantly higher in the gymnasts compared with the sedentary girls (0.23 (SE 0.01$) v .0 .29$ (SE 0.015$)$; $P<0.003$ ), whilst serum hsCRP concentrations (mg/L) were significantly lower $(0.49$ (SE 0.03$) v .1 .38$ (SE 0.19$)$; $P<0.0003)$. These differences remained significant after adjustment for body weight and height (analysis of covariance; $P<0.005$ ). Weight, height, BMI and dietary factors were all significantly lower in gymnasts compared with controls, whilst PA was significantly higher in gymnasts $(P<0.05$ for all variables). Among the gymnasts serum SICAM was inversely related to dietary total fat, MUFA and SFA (Table), but not to any anthropometric measures or levels of PA (by univariate analysis). Serum hsCRP was not significantly related to any of the variables.

\begin{tabular}{lccr}
\hline & \multicolumn{2}{c}{ sICAM } & hsCRP \\
\cline { 2 - 4 } Variables & Controls & Gymnasts & Controls \\
\hline Height $(\mathrm{m})$ & -0.250 & -0.378 & -0.076 \\
Wt $(\mathrm{kg})$ & -0.246 & -0.373 & 0.105 \\
BMI (kg/m ${ }^{2}$ ) & -0.113 & -0.119 & 0.382 \\
PA (min/week) & -0.080 & -0.187 & -0.105 \\
Dietary factors: & & & 0.231 \\
Energy intake (MJ) & 0.287 & -0.456 & 0.250 \\
Fat (g) & 0.456 & $-0.534^{*}$ & -0.096 \\
SFA (g) & 0.373 & $-0.549 *$ & -0.110 \\
MUFA (g) & 0.378 & $-0.487 *$ & -0.144 \\
PUFA (g) & 0.136 & -0.398 & -0.236 \\
CHO (g) & 0.001 & -0.355 & 0.117 \\
\hline
\end{tabular}

CHO, carbohydrate. $* P<0.05$.

The lower hsCRP levels in gymnasts may be related to their lower adiposity, although there was no significant correlation between BMI and hsCRP in either group. The higher concentrations of sICAM-1 in gymnasts may be related to the potential beneficial effects of intensive PA on endothelial function. Further analysis of skinfold thickness is underway.

The gymnast study was funded by the National Osteoporosis Society. E. A. is grateful to the Ministry of Health in Saudi Arabia for PhD funding.

1. Timpson NJ, Lawlor DA, Harbord RM et al. (2005) Lancet 366, 1954-1959.

2. Lo SK, Janakidevi K, Lai L et al. (1993) Am J Physiol 264, L406-L412.

3. Nemet D, Mills PJ \& Cooper DM (2004) Br J Sports Med 38, 154-158.

4. Giugliano D, Nappo F \& Coppola L (2001) Circulation 104, 34-35.

5. Nurmi-Lawton JA, Baxter-Jones AD, Mirwald RL et al. (2004) J Bone Miner Res 19, 314-322. 